\title{
Twist Quantization of String and Hopf Algebraic Symmetry ${ }^{\star}$
}

\author{
Tsuguhiko ASAKAWA and Satoshi WATAMURA \\ Department of Physics, Graduate School of Science, Tohoku University, \\ Aoba-ku, Sendai 980-8578, Japan \\ E-mail: asakawa@tuhep.phys.tohoku.ac.jp, watamura@tuhep.phys.tohoku.ac.jp
}

Received April 07, 2010, in final form August 12, 2010; Published online August 20, 2010 doi:10.3842/SIGMA.2010.068

\begin{abstract}
We describe the twist quantization of string worldsheet theory, which unifies the description of quantization and the target space symmetry, based on the twisting of Hopf and module algebras. We formulate a method of decomposing a twist into successive twists to analyze the twisted Hopf and module algebra structure, and apply it to several examples, including finite twisted diffeomorphism and extra treatment for zero modes.
\end{abstract}

Key words: string theory; qunatization; Hopf algebra; Drinfeld twist

2010 Mathematics Subject Classification: 83E30; 81T75; 53D55

\section{Introduction}

String theory is a promising candidate as a unified theory of elementary particles and quantum gravity. There are many evidences that string theory contains quantum gravity, the existence of graviton in the spectrum for example, and the fact that the conformal symmetry in the nonlinear sigma-model leads to the Einstein equation. However, it is not yet clear how the concepts of general relativity, such as general covariance or equivalence principle, are realized in string theory. The origin of these difficulties is that the theory is formulated only as a first quantized theory in a fixed background, and it is difficult to consider a change to other backgrounds.

In [1] and [2], we proposed a new formulation of string worldsheet theory based on the Hopf algebra structure which is minimally background dependent. In [1], we gave a unified formulation of the quantization of the string and the spacetime symmetry, by reformulating the path-integral quantization of the string as a Drinfeld twist [3] of the Hopf algebra. By the twist, the space-time diffeomorphism was deformed into a twisted Hopf algebra, while the Poincaré symmetry was kept unchanged. The method was applied to the constant $B$-field background in [2] and the structure of the twisted Hopf algebra in that case was studied. As a quantization, it implies a new normal ordering, and as a symmetry, we have shown that the twisted Poincaré symmetry $[4,5,6]$ in the noncommutative field theory on the Moyal plane are derived from our twisted Hopf algebra structure.

One of purposes of this paper is to give a overview on the twist quantization developed in $[1,2]$ so far. Part of the paper's results was previously presented at the workshop on Quantum Gravity and Quantum Geometry (Corfu, 2009) and published in its proceedings [7]. We repeat this material here for completeness' sake and for readers' convenience. We then formulate the method of decomposing a twist in a more general setting by extending [2], and discuss that it has wide variety of applications. New results of this paper include an improvement for interpretation

\footnotetext{
*This paper is a contribution to the Special Issue "Noncommutative Spaces and Fields". The full collection is available at http://www.emis.de/journals/SIGMA/noncommutative.html
} 
of the twisted diffeomorphism from the viewpoint of vertex operators, and the treatment of the zero modes for momentum conserving vacuum.

The paper is organized as follows: In Sections 2 and 3, we give a brief review of the twist quantization of string theory [1]. In Section 4, we describe a method of splitting the twist in great generality. Then, in Section 5, we give three examples applying the method, including the case for a constant $B$-field background [2] and two new examples. Section 6 is devoted to discussion and conclusion.

\section{Classical Hopf algebra in string theory}

We start with describing the classical Hopf algebra $\mathcal{H}$ of functional derivatives and its module algebra $\mathcal{A}$ of string functionals [1]. They depend on a target space manifold $M$ (here we fix $M=\mathbb{R}^{d}$ ) but are independent of the background data such as the metric and $B$ field.

The basic variable of the string worldsheet theory is a set of functions $X^{\mu}(z)(\mu=0, \ldots, d-1)$, or equivalently, a map $X: \Sigma \rightarrow M$ from the worldsheet $\Sigma$ into a target space $M$. We denote the space of such maps as $\mathcal{X}$. Let $\mathcal{A}=C(\mathcal{X})$ be the vector space of functionals $\mathcal{A} \ni I: X \mapsto I[X] \in \mathbb{C}$ of the abstract form

$$
I[X]=\int d^{2} z \rho(z) F[X(z)]
$$

where $F[X(z)] \in C^{\infty}(\Sigma)$ is given from a tensor field in the target space by pull-back, and $\rho(z)$ is some weight function (distribution). In particular, we call

$$
F[X]\left(z_{i}\right)=\int d^{2} z \delta^{(2)}\left(z-z_{i}\right) F[X(z)]
$$

a local functional at $z_{i}$, with an additional label $z_{i} \in \Sigma$. These functionals (2.1) and (2.2) correspond to an integrated vertex operator and to a local vertex operator after quantization, respectively. For example, for a one-form $\Omega=\Omega_{\mu}(x) d x^{\mu}$ in the target space we can define a functional

$$
I_{\Omega}[X]=\int d^{2} z \rho^{a}(z) \partial_{a} X^{\mu} \Omega_{\mu}[X(z)] .
$$

$\mathcal{A}$ forms a commutative algebra over $\mathbb{C}$, by defining a multiplication of two functionals $m$ : $\mathcal{A} \otimes \mathcal{A} \rightarrow \mathcal{A}$ as $(I J)[X]=I[X] J[X]$

Let $\mathfrak{X}$ be the space of all functional vector fields of the form

$$
\xi=\int d^{2} w \xi^{\mu}(w) \frac{\delta}{\delta X^{\mu}(w)}
$$

and denote its action on $I[X] \in \mathcal{A}$ as $\xi \triangleright I[X]$. Here $\xi^{\mu}(w)$ is a weight function (distribution) on the worldsheet including the following two classes.

i) $\xi^{\mu}(w)$ is a pull-back of a target space function $\xi^{\mu}(w)=\xi^{\mu}[X(w)]$. It is related to the variation of the functional under diffeomorphism $X^{\mu}(z) \rightarrow X^{\mu}(z)+\xi^{\mu}[X(z)]$ as $\delta_{\xi} F[X]=$ $-\xi \triangleright F[X]$. Note that it is arranged so that $\left(\xi \triangleright I_{\Omega}\right)[X]=I_{\mathcal{L}_{\xi} \Omega}[X]$ for a functional $(2.3)$, where $\mathcal{L}_{\xi}$ is the Lie derivative acting on the target space one-form $\Omega$.

ii) $\xi^{\mu}(w)$ is a function of $w$ but independent of $X(w)$ and its derivatives. It corresponds to a change of the embedding, $X^{\mu}(z) \rightarrow X^{\mu}(z)+\xi^{\mu}(z)$, and is used to derive the equation of motion. 
By successive transformations, $\xi \triangleright(\eta \triangleright F)$, the vector fields $\xi$ and $\eta$ form a Lie algebra with the Lie bracket

$$
[\xi, \eta]=\int d^{2} w\left(\xi^{\mu} \frac{\delta \eta^{\nu}}{\delta X^{\mu}}-\eta^{\mu} \frac{\delta \xi^{\nu}}{\delta X^{\mu}}\right)(w) \frac{\delta}{\delta X^{\nu}(w)} .
$$

Once we have a Lie algebra $\mathfrak{X}$, we can define its universal enveloping algebra $\mathcal{H}=U(\mathfrak{X})$ over $\mathbb{C}$, which has a natural cocommutative Hopf algebra structure $(U(\mathfrak{X}) ; \mu, \iota, \Delta, \epsilon, S)^{1}$. The defining maps given on elements $k \in \mathbb{C}$ and $\xi, \eta \in \mathfrak{X}$ are

$$
\begin{array}{lll}
\mu(\xi \otimes \eta)=\xi \cdot \eta, \quad \Delta(\xi)=\xi \otimes 1+1 \otimes \xi, & \epsilon(\xi)=0, & S(\xi)=-\xi, \\
\iota(k)=k \cdot 1, \quad \Delta(1)=1 \otimes 1, \quad \epsilon(1)=1, & S(1)=1 .
\end{array}
$$

As usual, these maps are uniquely extended to any element of $U(\mathfrak{X})$ by the algebra (anti-) homomorphism. There are many subalgebras in $\mathfrak{X}$. In particular, the Poincaré-Lie algebra $\mathcal{P}$ generated by

$$
P^{\mu}=-i \int d^{2} z \eta^{\mu \lambda} \frac{\delta}{\delta X^{\lambda}(z)}, \quad L^{\mu \nu}=-i \int d^{2} z X^{[\mu}(z) \eta^{\nu] \lambda} \frac{\delta}{\delta X^{\lambda}(z)},
$$

where $P^{\mu}$ are the generators of the translation and $L^{\mu \nu}$ are the Lorentz generators, forms a Hopf subalgebra of $\mathcal{H}=U(\mathfrak{X})$, denoted as $U(\mathcal{P})$. We denote the Abelian Lie subalgebra consisting of $\xi(2.4)$ in class ii) as $\mathfrak{C}$. Then $U(\mathfrak{C})$ is also a Hopf subalgebra of $\mathcal{H}$.

The algebra $\mathcal{A}$ of functionals is now considered as a $\mathcal{H}$-module algebra. The action on the product of two elements $F, G \in \mathcal{A}$ is a natural generalization of the action of $\xi$, defined by

$$
h \triangleright m(F \otimes G)=m \Delta(h) \triangleright(F \otimes G),
$$

satisfying the Leibniz rule $(\Delta(\xi)$ in $(2.5))$.

\section{Twist quantization}

In [1] we gave a simple quantization procedure in terms of a Hopf algebra twist, in which the vacuum expectation value (VEV) coincides with the conventional path integral average. A general theory of Hopf algebra twist is presented in [3, 9].

Suppose that there is a twist element (counital 2-cocycle), $\mathcal{F} \in \mathcal{H} \otimes \mathcal{H}$, which is invertible, counital $(\mathrm{id} \otimes \epsilon) \mathcal{F}=1$ and satisfies the 2-cocycle condition

$$
(\mathcal{F} \otimes \mathrm{id})(\Delta \otimes \mathrm{id}) \mathcal{F}=(\mathrm{id} \otimes \mathcal{F})(\mathrm{id} \otimes \Delta) \mathcal{F}
$$

Given a twist element $\mathcal{F}$, the twisted Hopf algebra $\mathcal{H}_{\mathcal{F}}$ can be defined by modifying the coproduct and antipode as

$$
\Delta_{\mathcal{F}}(h)=\mathcal{F} \Delta(h) \mathcal{F}^{-1}, \quad S_{\mathcal{F}}(h)=U S(h) U^{-1}
$$

for all $h \in \mathcal{H}$, where $U=\mu($ id $\otimes S) \mathcal{F}$. We regard this procedure of twisting as a quantization with respect to the twist element $\mathcal{F}$.

Correspondingly, the consistency of the action, i.e. covariance with respect to the Hopf algebra action, requires that a $\mathcal{H}$-module algebra $\mathcal{A}$ is twisted to the $\mathcal{H}_{\mathcal{F}}$-module algebra $\mathcal{A}_{\mathcal{F}}$. As a vector space, $\mathcal{A}_{\mathcal{F}}$ is identical to $\mathcal{A}$, but is accompanied by the twisted product

$$
F *_{\mathcal{F}} G=m_{\mathcal{F}}(F \otimes G):=m \circ \mathcal{F}^{-1} \triangleright(F \otimes G),
$$

\footnotetext{
${ }^{1}$ A similar Hopf algebra based on functional derivatives is also considered in [8].
} 
which is associative owing to the cocycle condition (3.1). The covariance means that for $h \in \mathcal{H}_{\mathcal{F}}$ and $F, G \in \mathcal{A}_{\mathcal{F}}$,

$$
h \triangleright m_{\mathcal{F}}(F \otimes G)=m \circ \Delta(h) \mathcal{F}^{-1} \triangleright(F \otimes G)=m_{\mathcal{F}} \Delta_{\mathcal{F}}(h) \triangleright(F \otimes G) .
$$

In this way the Hopf algebra and the module algebra are twisted in a consistent manner. The resulting twisted action is considered as a transformation in the quantized theories.

We define the vacuum expectation value $(\mathrm{VEV})$ for the twisted module algebra $\mathcal{A}_{\mathcal{F}}$ as the map $\tau: \mathcal{A}_{\mathcal{F}} \rightarrow \mathbb{C}$. For any element $I[X] \in \mathcal{A}_{\mathcal{F}}$ it is defined as the evaluation at $X=0$ :

$$
\tau(I[X]):=\left.I[X]\right|_{X=0} .
$$

If $I[X]$ is a product of two elements $F, G \in \mathcal{A}_{\mathcal{F}}$, it gives a correlation function

$$
\sigma(z, w)=\tau\left(F[X(z)] *_{\mathcal{F}} G[X(w)]\right)=\tau \circ m \circ \mathcal{F}^{-1} \triangleright(F \otimes G) .
$$

Owing to the associativity, the correlation function of $n$ local functionals is similarly given as

$$
\sigma\left(z_{1}, \ldots, z_{n}\right)=\tau\left(F_{1}\left[X\left(z_{1}\right)\right] * \mathcal{F} F_{2}\left[X\left(z_{2}\right)\right] \cdots * \mathcal{F} F_{n}\left[X\left(z_{n}\right)\right]\right) .
$$

The action of $h \in \mathcal{H}_{\mathcal{F}}$ inside the $\operatorname{VEV} \tau(I[X])$ is

$$
\tau(h \triangleright I[X]) .
$$

This action appears in the various relations related with the symmetry transformation.

This completes the description of the procedure. A different choice of a twist element $\mathcal{F}$ gives a quantization in a different background.

\subsection{Normal ordering and path integrals}

Here we assume that a twist element is in the Abelian Hopf subalgebra $\mathcal{F} \in U(\mathfrak{C}) \otimes U(\mathfrak{C})$ of the form

$$
\mathcal{F}=\exp \left(-\int d^{2} z \int d^{2} w G^{\mu \nu}(z, w) \frac{\delta}{\delta X^{\mu}(z)} \otimes \frac{\delta}{\delta X^{\nu}(w)}\right),
$$

specified by a Green function $G^{\mu \nu}(z, w)$ on the worldsheet $\Sigma$. It is easy to show that this $\mathcal{F}$ in (3.3) satisfies all conditions for twist elements [1]. In this case, the twist quantization described in the previous subsection is identical with the path integral quantization.

As proved in [1], the twist element $\mathcal{F}$ in (3.3) can be written as

$$
\mathcal{F}=\left(\mathcal{N}^{-1} \otimes \mathcal{N}^{-1}\right) \Delta(\mathcal{N}),
$$

where the normal ordering element $\mathcal{N} \in \mathcal{H}$ is defined by

$$
\mathcal{N}=\exp \left\{-\frac{1}{2} \int d^{2} z \int d^{2} w G^{\mu \nu}(z, w) \frac{\delta}{\delta X^{\mu}(z)} \frac{\delta}{\delta X^{\nu}(w)}\right\}
$$

This shows that the twist element $\mathcal{F}$ is a coboundary and thus it is trivial in the Hopf algebra cohomology. Consequently, there are isomorphisms summarized as:

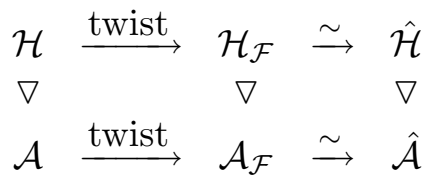


In the diagram, the left column is the classical pair $(\mathcal{H}, \mathcal{A})$, and the middle and the right column are the quantum counterparts. Here the map $\mathcal{H}_{\mathcal{F}} \stackrel{\sim}{\rightarrow} \hat{\mathcal{H}}$ is given by an inner automorphism $h \mapsto \mathcal{N} h \mathcal{N}^{-1} \equiv \tilde{h}$, and the map $\mathcal{A}_{\mathcal{F}} \stackrel{\sim}{\rightarrow} \hat{\mathcal{A}}$ is given by $F \mapsto \mathcal{N} \triangleright F \equiv: F:$ We call $\hat{\mathcal{H}}(\hat{\mathcal{A}})$ the normal ordered Hopf algebra (module algebra), respectively.

The normal ordered module algebra $\hat{\mathcal{A}}$ is the one which appears inside the VEV in the path integral. It consists of elements in normal ordered form

$$
\mathcal{N} \triangleright I[X] \equiv: I[X]:
$$

for any functional $I[X] \in \mathcal{A}_{\mathcal{F}}$. These elements correspond to vertex operators in the path integral, because the action of $\mathcal{N}$ (3.5) corresponds to subtractions of divergences at coincident points caused by self-contractions in the path integral. As an algebra, $\hat{\mathcal{A}}$ has the same multiplication $m: \hat{\mathcal{A}} \otimes \hat{\mathcal{A}} \rightarrow \hat{\mathcal{A}}$ as the classical functional $\mathcal{A}$. This is seen by the map (3.6) of the product in $\mathcal{A}_{\mathcal{F}}$ as

$$
\mathcal{N} \triangleright m \circ \mathcal{F}^{-1} \triangleright(F \otimes G)=m \circ(\mathcal{N} \otimes \mathcal{N}) \triangleright(F \otimes G),
$$

which is a direct consequence of the coboundary relation (3.4). An equivalent but more familiar expression is the time ordered product of two vertex operators as $:\left(F *_{\mathcal{F}} G\right):=: F:: G:$.

The normal ordered Hopf algebra $\hat{\mathcal{H}}$ is defined with the same algebraic operations as the classical Hopf algebra $\mathcal{H}$, but with elements dressed with a normal ordering as

$$
\mathcal{N} h \mathcal{N}^{-1} \equiv \tilde{h}
$$

for any $h \in \mathcal{H}_{\mathcal{F}}$. The isomorphism map (3.6) relates the twisted Hopf algebra action $\mathcal{H}_{\mathcal{F}}$ on $\mathcal{A}_{\mathcal{F}}$ to the corresponding action of $\hat{\mathcal{H}}$ on $\hat{\mathcal{A}}$. For example,

$$
\begin{aligned}
& h \triangleright F \stackrel{\sim}{\longrightarrow} \mathcal{N} \triangleright(h \triangleright F)=\tilde{h} \triangleright: F:, \\
& h \triangleright(F * \mathcal{F} G) \stackrel{\sim}{\longrightarrow} \tilde{h} \triangleright(: F:: G:) .
\end{aligned}
$$

The elements in $\hat{\mathcal{A}}$ contain generically formal divergent series (this is the reason why we distinguish $\mathcal{A}$ and $\hat{\mathcal{A}}$ ), and thus equations (3.7) and (3.8) have only a meaning inside the VEV [1]. The VEV (3.2) for $\mathcal{A}_{\mathcal{F}}$ implies the definition of the VEV for $\hat{\mathcal{A}}$ to be a map, $\tau \circ \mathcal{N}^{-1}: \hat{\mathcal{A}} \rightarrow \mathbb{C}$, and it turns out that it coincides with the $\operatorname{VEV}\langle\cdots\rangle$ in the path integral

$$
\langle\mathcal{O}\rangle:=\frac{\int \mathcal{D} X \mathcal{O} e^{-S}}{\int \mathcal{D} X e^{-S}} .
$$

For instance, the correlation of two local functionals is

$$
\tau \circ \mathcal{N}^{-1} \triangleright(: F[X]:(z): G[X]:(w))=\langle: F[X]:(z): G[X]:(w)\rangle
$$

The equality (3.10) can be easily verified using the standard path integral argument (see for example [10]). Clearly, the action functional $S[X]$ in (3.9) is related to the Green function in $\mathcal{F}$ and $\mathcal{N}$. It is quadratic $S[X]=\frac{1}{2} \int d^{2} z X^{\mu} D_{\mu \nu} X^{\nu}$ with a fixed second order derivative $D_{\mu \nu}$ such that the Green function in (3.5) is a solution of the equation $D_{\mu \rho} G^{\rho \nu}(z, w)=\delta_{\mu}^{\nu} \delta^{(2)}(z-w)$. This explains why we call a twist element $\mathcal{F}$ a background in string worldsheet theory. Here, the action $\mathcal{N}^{-1}$ gives the Wick contraction with respect to the Green function. Note that in (3.9), each local insertion is understood as being regulated by the (conformal) normal ordering $\mathcal{N}$.

The two descriptions in terms of a twisted pair $\left(\mathcal{H}_{\mathcal{F}}, \mathcal{A}_{\mathcal{F}}\right)$ and of a normal ordered pair $(\hat{\mathcal{H}}, \hat{\mathcal{A}})$ (thus path integral) are equivalent. However, note that the background dependences in the two formulations are different. In the case of the normal ordered pair $(\hat{\mathcal{H}}, \hat{\mathcal{A}})$, both an element $: F: \in \hat{\mathcal{A}}$ and the VEV $\tau \circ \mathcal{N}^{-1}$ contain $\mathcal{N}$ which depends on the background. In the operator formulation this corresponds to the property that a mode expansion of the string variable $X^{\mu}(z)$ as well as the oscillator vacuum are background dependent. Therefore, the description of the quantization that makes $\hat{\mathcal{A}}$ well-defined is only applicable to that particular background and we need a different mode expansion for a different background. 


\subsection{Twisted Hopf algebraic symmetry}

One advantage of the twist quantization is that both, the module algebra $\mathcal{A}$ (observables) and the Hopf algebra $\mathcal{H}$ (symmetry) are simultaneously quantized by a single twist element $\mathcal{F}$ (background). This gives us a good understanding of the symmetry structure after the quantization, as discussed in [1] in great detail: There, we argued that the symmetry of the theory in the conventional sense, namely the background preserving diffeomorphism, is characterized as a twist invariant Hopf subalgebra of $\mathcal{H}_{\mathcal{F}}$, which consists of elements $h \in \mathcal{H}$ such that $\Delta_{\mathcal{F}}(h)=\Delta(h)$ and $S_{\mathcal{F}}(h)=S(h)$. Of course, this subalgebra depends on the choice of $\mathcal{F}$ (background). The other elements of $\mathcal{H}_{\mathcal{F}}$, i.e., generic diffeomorphisms, should be twisted at the quantum level.

To be more precise, let us fix a twist element to be the Minkowski background with the metric $\eta_{\mu \nu}$. We denote it as $\mathcal{F}_{0}$. It is achieved by setting the Green function in (3.3) for that background. For instance, on the worldsheet $\Sigma=\mathbb{C}$, it is given by

$$
G_{0}^{\mu \nu}(z, w)=-\alpha^{\prime} \eta^{\mu \nu} \ln |z-w| .
$$

In this case, the universal enveloping algebra $U(\mathcal{P})$ (2.6) for the Poincaré Lie algebra $\mathcal{P}$ is the true (quantum) symmetry of the theory. It is easily checked that the twist does not alter the coproduct $\Delta_{\mathcal{F}_{0}}(u)=\Delta(u)$ nor the antipode $S_{\mathcal{F}_{0}}(u)=S(u)$ for $\forall u \in U(\mathcal{P})$ and thus $U(\mathcal{P})$ is identical with the original $U(\mathcal{P})$ under the twisting by $\mathcal{F}_{0}$. Therefore, the twist quantization coincides with the ordinary quantization, in which the Poincaré covariance is assumed to hold at the quantum level.

We have not yet fully understood the structure of the twisted diffeomorphism around this background, in particular from the target-space viewpoint. But it should contain the information on the broken diffeomorphism. There is an argument that the action of this twisted diffeomorphism can be regarded as a remnant of the classical diffeomorphism, where the change of the background under diffeomorphisms is incorporated into the twisted diffeomorphism in such a way that the twist element (quantization scheme) is kept invariant. A similar statement in the case of twisted diffeomorphism on the Moyal plane is found in [11]. We will come back to this point below.

\section{Decomposition of twists}

In this section, we extend the method discussed in [2] and describe a general theory of decomposing a twist into successive twists, which is a useful tool to relate two different backgrounds.

We will consider two different twist elements $\mathcal{F}_{0}$ and $\mathcal{F}_{1}$ in $\mathcal{H}$, that are related by

$$
\mathcal{F}_{1}=\mathcal{F}_{d} \mathcal{F}_{0}
$$

for some $\mathcal{F}_{d}$. We assume that the first twist $\mathcal{F}_{0}$ is given by the propagator $G_{0}$ as $(3.3)$, which defines a quantization scheme (background) as described in the previous section. In general, for a given twisted Hopf algebra $\mathcal{H}_{\mathcal{F}_{0}}$ with a coproduct $\Delta_{\mathcal{F}_{0}}$, a further twisting by $\mathcal{F}_{d}$ is possible if $\mathcal{F}_{d}$ is a twist element in $\mathcal{H}_{\mathcal{F}_{0}}$ (not in $\mathcal{H}$ )

$$
\left(\mathcal{F}_{d} \otimes \mathrm{id}\right)\left(\Delta_{\mathcal{F}_{0}} \otimes \mathrm{id}\right) \mathcal{F}_{d}=\left(\mathrm{id} \otimes \mathcal{F}_{d}\right)\left(\mathrm{id} \otimes \Delta_{\mathcal{F}_{0}}\right) \mathcal{F}_{d}
$$

Then, $\mathcal{F}_{1}=\mathcal{F}_{d} \mathcal{F}_{0}$ is also a twist element in $\mathcal{H}$. This is because the 1.h.s. of the 2-cocycle condition (3.1) for $\mathcal{F}_{1}$ can be written as

$$
\left(\mathcal{F}_{1} \otimes \mathrm{id}\right)(\Delta \otimes \mathrm{id}) \mathcal{F}_{1}=\left[\left(\mathcal{F}_{d} \otimes \mathrm{id}\right)\left(\Delta_{\mathcal{F}_{0}} \otimes \mathrm{id}\right) \mathcal{F}_{d}\right]\left[\left(\mathcal{F}_{0} \otimes \mathrm{id}\right)(\Delta \otimes \mathrm{id}) \mathcal{F}_{0}\right]
$$

and similarly for the r.h.s., where (3.1) for $\mathcal{F}_{0}$ is used. Then by using (4.2), it is shown that $\mathcal{F}_{1}$ satisfies (3.1). Conversely, if two twist elements $\mathcal{F}_{0}$ and $\mathcal{F}_{1}$ in $\mathcal{H}$ have a relation $\mathcal{F}_{1}=\mathcal{F}_{d} \mathcal{F}_{0}$, 
then $\mathcal{F}_{d}$ is a twist element in $\mathcal{H}_{\mathcal{F}_{0}}$ satisfying (4.2). In fact, the decomposition (4.1) defines two successive twists of the Hopf algebra $\mathcal{H}$ and the module algebra $\mathcal{A}$ :

$$
(\mathcal{H}, \mathcal{A}) \stackrel{\text { twist by } \mathcal{F}_{0}}{\longrightarrow}\left(\mathcal{H}_{\mathcal{F}_{0}}, \mathcal{A}_{\mathcal{F}_{0}}\right) \stackrel{\text { twist by } \mathcal{F}_{d}}{\longrightarrow}\left(\mathcal{H}_{\mathcal{F}_{1}}, \mathcal{A}_{\mathcal{F}_{1}}\right)
$$

This means that the identities $\left(\Delta_{\mathcal{F}_{0}}\right)_{\mathcal{F}_{d}}=\Delta_{\mathcal{F}_{1}}$ and $\left(S_{\mathcal{F}_{0}}\right)_{\mathcal{F}_{d}}=S_{\mathcal{F}_{1}}$ hold for coproducts and antipodes (see Appendix for the proof).

The main advantage to decompose a twist element is that we can use the isomorphism $\mathcal{A}_{\mathcal{F}_{0}} \simeq \hat{\mathcal{A}}_{0}$ (3.6). Then, the second twist by $\mathcal{F}_{d}$ in (4.3) can also be regarded as a twist of the normal ordered module algebra (vertex operators). This enables us to study the effect of a change of background in terms of vertex operators, as we will see.

First, the twisted product of two local functional $F, G \in \mathcal{A}_{\mathcal{F}_{1}}$ is rewritten by using (4.1) as

$$
\begin{aligned}
F * \mathcal{F}_{1} G & =m \circ \mathcal{F}_{1}^{-1} \triangleright(F \otimes G)=m \circ \Delta\left(\mathcal{N}_{0}^{-1}\right)\left(\mathcal{N}_{0} \otimes \mathcal{N}_{0}\right) \mathcal{F}_{d}^{-1} \triangleright(F \otimes G) \\
& =\mathcal{N}_{0}^{-1} \triangleright m \circ \tilde{\mathcal{F}}_{d}^{-1}\left(\mathcal{N}_{0} \triangleright F \otimes \mathcal{N}_{0} \triangleright G\right)=\mathcal{N}_{0}^{-1} \triangleright\left(: F: *_{\tilde{\mathcal{F}}_{d}}: G:\right),
\end{aligned}
$$

where the coboundary relation (3.4) for $\mathcal{F}_{0}^{-1}$ is used. Note that the dressed version of the twist element, defined by $\tilde{\mathcal{F}}_{d}=\left(\mathcal{N}_{0} \otimes \mathcal{N}_{0}\right) \mathcal{F}_{d}\left(\mathcal{N}_{0}^{-1} \otimes \mathcal{N}_{0}^{-1}\right) \in \hat{\mathcal{H}}_{0} \otimes \hat{\mathcal{H}}_{0}$ appears in the expression, because it should act on $\hat{\mathcal{A}}_{0} \otimes \hat{\mathcal{A}}_{0}$. In fact, it is shown that $\tilde{\mathcal{F}}_{d}$ is a twist element in $\hat{\mathcal{H}}_{0}$. In the same manner, the action of $h \in \mathcal{H}_{\mathcal{F}_{1}}$ on the same product is written as

$$
h \triangleright\left(F * \mathcal{F}_{1} G\right)=\mathcal{N}_{0}^{-1} \tilde{h} \triangleright\left(: F: *_{\tilde{\mathcal{F}}_{d}}: G:\right)=\mathcal{N}_{0}^{-1} \triangleright m \circ \tilde{\mathcal{F}}_{d}{ }^{-1} \Delta_{\tilde{\mathcal{F}}_{d}}(\tilde{h}) \triangleright(: F: \otimes: G:),
$$

where again $\tilde{h}=\mathcal{N}_{0} h \mathcal{N}_{0}^{-1}$ acts on $\hat{\mathcal{A}}_{0}$. These relations (4.4) and (4.5) show that $\mathcal{A}_{\mathcal{F}_{1}}$ is isomorphic to the twisted module algebra $\left(\hat{\mathcal{A}}_{0}\right)_{\tilde{\mathcal{F}}_{d}}$ under the map $F \rightarrow: F$ : by the $\mathcal{N}_{0}$ action. The structure is summarized as

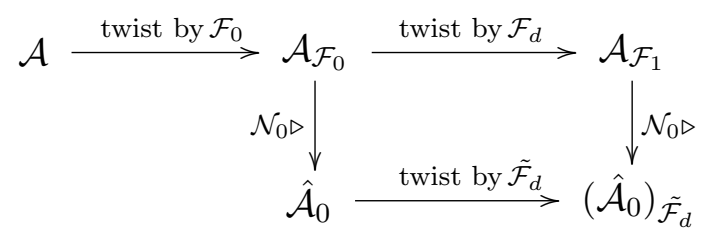

The VEV for $\mathcal{A}_{\mathcal{F}_{1}}$ is also written in terms of $\left(\hat{\mathcal{A}}_{0}\right)_{\tilde{\mathcal{F}}_{d}}$ as

$$
\tau\left(F *_{\mathcal{F}_{1}} G\right)=\tau \circ \mathcal{N}_{0}^{-1} \triangleright\left(: F: *_{\tilde{\mathcal{F}}_{d}}: G:\right)=\left\langle: F: *_{\tilde{\mathcal{F}}_{d}}: G:\right\rangle_{0} .
$$

Thus the effect of the second twisting by $\mathcal{F}_{d}$ is completely incorporated as the twisting of the normal ordered Hopf algebra $\hat{\mathcal{H}}_{0}$ and module algebra $\hat{\mathcal{A}}_{0}$. It means that under the decomposition (4.1), the first twist $\mathcal{F}_{0}$ serves as a quantization w.r.t. a fixed background, and the second twist $\mathcal{F}_{d}$ play the role of the deformation of the vertex operator product with keeping the background.

\subsection{Relating two backgrounds}

The above procedure is possible for any $\mathcal{F}_{d}$ if it exists. Let us now specialize the situation, in which $\mathcal{F}_{1}$ has also the form (3.3) for some propagator $G_{1}$. Then it also defines a quantization scheme, which is achieved by the twist element as in (3.3)

$$
\mathcal{F}_{1}:=\exp \left\{-\int d^{2} z \int d^{2} w G_{1}^{\mu \nu}(z, w) \frac{\delta}{\delta X^{\mu}(z)} \otimes \frac{\delta}{\delta X^{\nu}(w)}\right\} .
$$


Namely, we obtain the twisted pair $\left(\mathcal{H}_{\mathcal{F}_{1}}, \mathcal{A}_{\mathcal{F}_{1}}\right)$ as well as the normal ordered pair $\left(\hat{\mathcal{H}}_{1}, \hat{\mathcal{A}}_{1}\right)$ of Hopf and module algebras as in Section 3. We denote this normal ordering as ${ }_{\circ}^{\circ} . . \circ$, then for example

$$
{ }_{\circ}^{\circ} F * \mathcal{F}_{1} G_{\circ}^{\circ}={ }_{\circ}^{\circ} F_{\circ \circ}^{\circ \circ} G_{\circ}^{\circ}
$$

holds.

From the assumption that both $\mathcal{F}_{0}$ and $\mathcal{F}_{1}$ has the form (3.3), the deviation $\mathcal{F}_{d}$ in (4.1) has necessarily the form (3.3). It is equivalent to the decomposition of the propagator

$$
G_{1}^{\mu \nu}(z, w)=G_{0}^{\mu \nu}(z, w)+G_{d}^{\mu \nu}(z, w)
$$

which is the situation discussed in [2]. It is now possible to relate $\hat{\mathcal{A}}_{1}$ and $\left(\hat{\mathcal{A}}_{0}\right)_{\tilde{\mathcal{F}}_{d}}$, and this gives an additional structure to (4.6) as follows. First, note that $\tilde{\mathcal{F}}_{d}=\mathcal{F}_{d}$ in this case. We denote two normal ordered functionals as $: F: \in \hat{\mathcal{A}}_{0}$ and ${ }_{\circ}^{\circ} F_{\circ}^{\circ} \in \hat{\mathcal{A}}_{1}$. According to (4.1), two normal orderings are also related as $\mathcal{N}_{1}=\mathcal{N}_{d} \mathcal{N}_{0}$. Then, we have a relation ${ }_{\circ}^{\circ} F_{\circ}^{\circ}=\mathcal{N}_{d} \triangleright: F$ : between them. The relation is such that the VEVs, each with respect to the corresponding quantization scheme, give the same result

$$
\left\langle{ }_{0}^{\circ} F_{\circ}^{\circ}\right\rangle_{1}=\tau\left(\mathcal{N}_{1}^{-1} \triangleright{ }_{\circ}^{\circ} F_{\circ}^{\circ}\right)=\tau\left(\mathcal{N}_{0}^{-1} \triangleright: F:\right)=\langle: F:\rangle_{0} .
$$

The difference of two quantization schemes lies in the Wick contraction of several local functionals. In fact, applying $\tau$ on both sides of (4.4) leads to the equation of the VEV

$$
\left\langle{ }_{0}^{\circ} F_{\circ \circ}^{\circ \circ} G_{\circ}^{\circ}\right\rangle_{1}=\tau\left(F * \mathcal{F}_{1} G\right)=\left\langle: F: * \mathcal{F}_{d}: G:\right\rangle_{0}
$$

On the l.h.s., the definition of the VEV as well as the normal ordering are with respect to the new quantization $\mathcal{F}_{1}$. The r.h.s. is written in terms of the original quantization scheme $\mathcal{F}_{0}$ except that the OPE is twisted by $\mathcal{F}_{d}$, that is $\left(\hat{\mathcal{A}}_{0}\right)_{\mathcal{F}_{d}}$ in $(4.6)$. Similarly, the twisted Hopf algebra $\left(\hat{\mathcal{H}}_{0}\right)_{\mathcal{F}_{d}}$ is mapped to the normal ordered Hopf algebra $\hat{\mathcal{H}}_{1}$ by $\tilde{h} \rightarrow \mathcal{N}_{d} \tilde{h} \mathcal{N}_{d}^{-1}=\mathcal{N}_{1} h \mathcal{N}_{1}^{-1} \equiv \tilde{h}_{1}$ and we have

$$
\left\langle\tilde{h}_{1} \triangleright{ }_{\circ}^{\circ} F_{\circ \circ}^{\circ \circ} G_{\circ}^{\circ}\right\rangle_{1}=\left\langle\tilde{h} \triangleright\left(: F: * \mathcal{F}_{d}: G:\right)\right\rangle_{0},
$$

Let us summarize the whole structure:

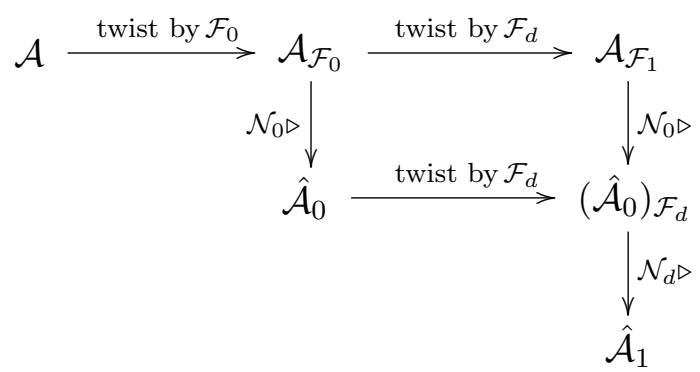

In this way, given two backgrounds $\mathcal{F}_{0}$ and $\mathcal{F}_{1}$, the corresponding quantum operator algebras and symmetries are related through the deformation $\mathcal{F}_{d}$, and since $\mathcal{F}_{1}=\mathcal{F}_{d} \mathcal{F}_{0}=\mathcal{F}_{0} \mathcal{F}_{d}$, we can also exchange the role of $\mathcal{F}_{0}$ and $\mathcal{F}_{d}$.

\section{Applications}

Here we apply the method given in Section 4 to three cases: the constant $B$-field background, target space diffeomorphism, and extra twist in zero modes. The first is already appeared in [2] and the others are new. 


\subsection{Twist quantization with $B$ field}

As an application of the above mentioned method, let us consider the background with a constant $B$-field. For simplicity, we take the worldsheet $\Sigma$ to be the upper half plane of the complex plane. Then, the propagator satisfying the mixed boundary condition is given as $[12,13,14]$

$$
G_{1}^{\mu \nu}(z, w)=-\alpha^{\prime}\left[\eta^{\mu \nu} \ln |z-w|-\eta^{\mu \nu} \ln |z-\bar{w}|+G^{\mu \nu} \ln |z-\bar{w}|^{2}+\Theta^{\mu \nu} \ln \frac{z-\bar{w}}{\bar{z}-w}\right],
$$

where the open string metric $G^{\mu \nu}$ and the antisymmetric tensor $\Theta^{\mu \nu}$ are defined by

$$
G^{\mu \nu}=\left(\frac{1}{\eta+B} \eta \frac{1}{\eta-B}\right)^{\mu \nu} \quad \text { and } \quad \Theta^{\mu \nu}=\frac{\theta^{\mu \nu}}{2 \pi \alpha^{\prime}}=-\left(\frac{1}{\eta+B} B \frac{1}{\eta-B}\right)^{\mu \nu} .
$$

We take the twist $\mathcal{F}_{1}$ of the form (3.3) with this propagator. Twisting by $\mathcal{F}_{1}$ directly, we obtain the normal ordered module algebra $\hat{\mathcal{A}}_{1}$. The same normal ordering in the context of operator formalism is given in the literature $[15,16,17,18]$. Here we decompose $\mathcal{F}_{1}$ in two different ways [2].

\subsubsection{Relation to field theory and twisted Poincaré symmetry}

The first decomposition is meaningful only for the open string case. By restricting the functional space to the one corresponding to the open string vertex operators, we can derive the relation to the twisted Poincare symmetry in the field theory on the noncommutative space considered in $[4,5,6]$.

To this end, we decompose the propagator into the symmetric part $G_{S}$ and the anti-symmetric part $G_{A}$ with respect to the tensor indices $\mu \nu$ :

$$
G_{1}^{\mu \nu}(z, w)=G_{S}^{\mu \nu}(z, w)+G_{A}^{\mu \nu}(z, w) .
$$

At the boundary $s, t \in \partial \Sigma=\mathbb{R}$, they reduce to the form

$$
G_{S}^{\mu \nu}(s, t)=-\alpha^{\prime} G^{\mu \nu} \ln (s-t)^{2} \quad \text { and } \quad G_{A}^{\mu \nu}(s, t)=\frac{i}{2} \theta^{\mu \nu} \epsilon(s-t),
$$

where $\epsilon(t)$ is the sign function. This implies the decomposition $\mathcal{F}_{1}=\mathcal{F}_{A} \mathcal{F}_{S}$ (4.1). Under the first twist, we have a twisted module algebra $\mathcal{A}_{\mathcal{F}_{S}}$ with the product $* \mathcal{F}_{S}$, and the normal ordered module algebra $\hat{\mathcal{A}}_{S}$ (see (4.8)). We denote elements of $\hat{\mathcal{A}}_{S}$ as $: F:=\mathcal{N}_{S} \triangleright F$. Clearly, this defines a quantization scheme w.r.t. the open string metric as a background, but it turns out that it is natural only for boundary elements of $\mathcal{H}$ and $\mathcal{A} .^{2}$

The quantum (untwisted) symmetry in this case is easily found. Although the Lorentz generator $L_{\mu \nu} \in \mathcal{P}$ in (2.6) acquire the twist and become non-primitive, there are other boundary elements $L_{\mu \nu}^{\prime} \in \mathcal{H}$ of the form

$$
L_{\mu \nu}^{\prime}=\int_{\partial \Sigma} d t G_{[\mu \rho} X^{\rho}(t) \frac{\delta}{\delta X^{\nu]}(t)}
$$

such that $L_{\mu \nu}^{\prime}$ and the translation generators $P_{\mu}$ (as boundary elements) constitute another Poincaré-Lie algebra $\mathcal{P}^{\prime}$ when acting on boundary local functionals, where the commutation relations are written with respect to the open string metric $G_{\mu \nu}$. It is easy to show that the Hopf subalgebra $U\left(\mathcal{P}^{\prime}\right)$ of $\mathcal{H}$ is invariant under the twist $\mathcal{F}_{S}$. In terms of the normal ordered Hopf algebra, this means $\tilde{P}_{\mu}=P_{\mu}$ and $\tilde{L}_{\mu \nu}^{\prime}=L_{\mu \nu}^{\prime}$ as boundary elements in $\hat{\mathcal{H}}_{S}$. Therefore, $U\left(\mathcal{P}^{\prime}\right)$ is

\footnotetext{
${ }^{2}$ They are defined by the functionals and functional derivatives of the embedding $X^{\mu}(t)$ of the boundary, or equivalently, defined by inserting a delta function of the form $\int_{\partial \Sigma} d t \delta^{(2)}(z-t)$ into $\mathcal{H}$ and $\mathcal{A}$.
} 
considered to be a quantum Poincaré symmetry in this quantization scheme when restricted on the boundary.

As a consequence, boundary elements of the module algebra $\hat{\mathcal{A}}_{S}$ are classified by the representation of $U\left(\mathcal{P}^{\prime}\right)$ with a fixed momentum $k_{\mu}$. In general, a local boundary vertex operator $V_{k}(t)$ with momentum $k_{\mu}$ consists of the worldsheet derivatives of $X$ and $e^{i k \cdot X}$, having the form

$$
V_{k}(t)=: P[\partial X(t)] e^{i k \cdot X(t)} \bullet
$$

Here $P[\partial X]$ denotes a polynomial. Note these vertex operators $V_{k}(t)$ are equivalent to the vertex operators used in [19], because their anomalous dimension and the on-shell condition are determined with respect to the open string metric $G_{\mu \nu}$.

Twisting by $\mathcal{F}_{A}$ further, we obtain the twisted Hopf algebra $\left(\hat{\mathcal{H}}_{S}\right)_{\mathcal{F}_{A}}$ and the twisted module algebra $\mathcal{A}_{\mathcal{F}_{1}} \simeq\left(\hat{\mathcal{A}}_{S}\right)_{\mathcal{F}_{A}}$ (see (4.8)). The twisted product $*_{\mathcal{F}_{1}}$ in $\mathcal{A}_{\mathcal{F}_{1}}$ is seen as a deformation of the product in $\hat{\mathcal{A}}_{S}$ as above:

$$
\left\langle{ }_{0}^{\circ} F_{\circ \circ}^{\circ} G_{\circ}^{\circ}\right\rangle_{1}=\tau\left(F * \mathcal{F}_{1} G\right)=\left\langle F_{\bullet}^{\bullet} * \mathcal{F}_{A} \bullet G_{\bullet}^{\bullet}\right\rangle_{S} .
$$

By considering only the boundary vertex operators, the twist element acting on them has the form

$$
\mathcal{F}_{A}=\exp \left\{-\frac{i}{2} \theta^{\mu \nu} \int d s \int d t \epsilon(s-t) \frac{\delta}{\delta X^{\mu}(s)} \otimes \frac{\delta}{\delta X^{\nu}(t)}\right\} .
$$

Because worldsheet derivatives of $X$ do not feel this deformation: e.g., $\partial_{a} X^{\mu}(s) * \mathcal{F}_{A} X^{\nu}(t)=$ $\partial_{a} X^{\mu}(s) X^{\nu}(t)$, for any correlation function of boundary vertex operators of the form (5.2), the product $*_{\mathcal{F}_{A}}$ is only sensitive to the exponential part, and we have

$$
\left\langle V_{k_{1}}\left(t_{1}\right) * \mathcal{F}_{A} \cdots * \mathcal{F}_{A} V_{k_{n}}\left(t_{n}\right)\right\rangle_{S}=e^{-\frac{i}{2} \sum_{i>j} k_{i \mu} \theta^{\mu \nu} k_{j \nu} \epsilon\left(t_{i}-t_{j}\right)}\left\langle V_{k_{1}}\left(t_{1}\right) \cdots V_{k_{n}}\left(t_{n}\right)\right\rangle_{S}
$$

In order to move from correlation functions on the worldsheet to the effective theory on Dbranes, we should fix the cyclic ordering of the insertion points $t_{1}>t_{2}>\cdots>t_{n}$ [19]. Then the extra phase factor in the r.h.s. above becomes independent of the precise locations, and gives the factor of the Moyal product. This enables us to take the prescription [19]: replace ordinary multiplication in the effective field theory written in the open string metric by the Moyal product. In the Hopf algebra language, this means that with a fixed ordering the twist element (5.3) acts as a Moyal-twist [20, 21]

$$
\mathcal{F}_{M}=e^{\frac{i}{2} \theta^{\mu \nu} P_{\mu} \otimes P_{\nu}},
$$

on fields on the D-brane. Here, the integrals of functional derivatives in (5.3) are replaced by its zero mode part, the translation generators $P_{\mu} \in U\left(\mathcal{P}^{\prime}\right)$.

The Poincaré symmetry on D-branes is also read off under the above prescription. The coproduct of the Lorentz generators $L_{\mu \nu}^{\prime} \in U\left(\mathcal{P}^{\prime}\right)$ acting on boundary vertex operators is twisted by $\mathcal{F}_{A}$ as

$$
\Delta_{\mathcal{F}_{A}}\left(L_{\mu \nu}^{\prime}\right)=\Delta\left(L_{\mu \nu}^{\prime}\right)+\frac{1}{2} \theta^{\alpha \beta} \int d s \int d t \epsilon(s-t) G_{\alpha[\mu} \frac{\delta}{\delta X^{\nu]}(s)} \otimes \frac{\delta}{\delta X^{\beta}(t)}
$$

but it reduces by fixing the ordering $s>t$ to

$$
\Delta_{\mathcal{F}_{M}}\left(L_{\mu \nu}^{\prime}\right)=\Delta\left(L_{\mu \nu}^{\prime}\right)+\frac{1}{2} \theta^{\alpha \beta}\left\{G_{\alpha[\mu} P_{\nu]} \otimes P_{\beta}+P_{\alpha} \otimes G_{\beta[\mu} P_{\nu]}\right\} .
$$

This is nothing but the twisted Poincaré-Hopf algebra $U_{\mathcal{F}_{M}}\left(\mathcal{P}^{\prime}\right)$ found in $[4,5,6]$. Note that since the twist element itself now belongs to $U\left(\mathcal{P}^{\prime}\right) \otimes U\left(\mathcal{P}^{\prime}\right)$, the twisting is closed in $U\left(\mathcal{P}^{\prime}\right)$. 
To summarize, the first twist is a quantization with respect to the open string metric, when restricted to boundary vertex operators, and the second is its deformation, which reduces to the Moyal-twist on the D-brane worldvolume. In other words, the twisted Poincare symmetry on the Moyal-Weyl noncommutative space is derived from string worldsheet theory in a $B$-field background. Thus, we establish the diagram schematically

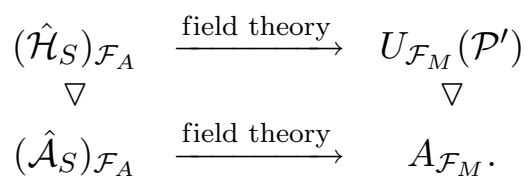

\subsection{2 $B$ field as a deformation}

The next decomposition is more natural from the viewpoint of the twist quantization. There we can see the relation between the standard quantization and the deformation caused by a $B$-field background. With this decomposition, we obtain a new twisted Poincaré symmetry, which is different from $[4,5,6]$ realized only on D-branes.

Here we consider a decomposition of the propagator (5.1) into the $B=0$ part and $B$ dependent part:

$$
\begin{aligned}
& G_{1}^{\mu \nu}(z, w)=G_{0}^{\mu \nu}(z, w)+G_{B}^{\mu \nu}(z, w), \\
& G_{B}^{\mu \nu}(z, w)=-\alpha^{\prime}\left[(G-\eta)^{\mu \nu} \ln |z-\bar{w}|^{2}+\Theta^{\mu \nu} \ln \frac{z-\bar{w}}{\bar{z}-w}\right],
\end{aligned}
$$

where $G_{0}^{\mu \nu}(z, w)$ is given in (3.11). Accordingly, the twist element $\mathcal{F}_{1}$ (4.7) is divided into

$$
\mathcal{F}_{1}=\mathcal{F}_{B} \mathcal{F}_{0}
$$

Here the effect of the $B$-field is completely characterized as a (second) twist $\mathcal{F}_{B}$ of the Hopf algebra $\mathcal{H}_{\mathcal{F}_{0}}$ and the module algebra $\mathcal{A}_{\mathcal{F}_{0}}$, that are regarded as a deformation of $\hat{\mathcal{H}}_{0}$ and the vertex operator algebra $\hat{\mathcal{A}}_{0}$ :

$$
\left\langle{ }_{\circ}^{\circ} F_{\circ \circ}^{\circ \circ} G_{\circ}^{\circ}\right\rangle_{1}=\left\langle: F: * \mathcal{F}_{B}: G:\right\rangle_{0} .
$$

Let us now focus on the fate of the Poincaré symmetry $U(\mathcal{P})$. Under the first twist, $U(\mathcal{P})$ is a twist invariant Hopf subalgebra of $\mathcal{H}_{\mathcal{F}_{0}}$. Equivalently, $U(\mathcal{P})$ is a Hopf subalgebra of $\hat{\mathcal{H}}_{0}$ with $\tilde{P}_{\mu}=P_{\mu}$ and $\tilde{L}_{\mu \nu}=L_{\mu \nu}$ as elements in $\hat{\mathcal{H}}_{0}$. Thus, $U(\mathcal{P})$ remains a symmetry at the quantum level, and each (normal ordered) vertex operator in $\hat{\mathcal{A}}_{0}$ is in a representation of a Poincaré-Lie algebra. We emphasize that this fact guarantees the spacetime meaning of a vertex operator. For example, a graviton vertex operator $V=: \partial X^{\mu} \bar{\partial} X^{\nu} e^{i k X}$ : transforms as spin 2 representation under $U(\mathcal{P})$, corresponding to the spacetime graviton field $h_{\mu \nu}(x)$.

However, in a $B$-field background, the second twist $\hat{\mathcal{H}}_{0} \rightarrow\left(\hat{\mathcal{H}}_{0}\right) \mathcal{F}_{B}$ modifies the coproduct and antipode of the Lorentz generator in $U(\mathcal{P})$ as

$$
\begin{aligned}
& \Delta_{\mathcal{F}_{B}}\left(L_{\mu \nu}\right)=L_{\mu \nu} \otimes 1+1 \otimes L_{\mu \nu}-2 \int d^{2} z d^{2} w \eta_{[\mu \alpha} G_{B}^{\alpha \beta}(z, w) \frac{\delta}{\delta X^{\beta}(z)} \otimes \frac{\delta}{\delta X^{\nu]}(z)}, \\
& S_{\mathcal{F}_{B}}\left(L_{\mu \nu}\right)=S\left(L^{\mu \nu}\right)+2 \int d^{2} u d^{2} z G_{B}^{\rho[\mu}(u, z) \frac{\delta}{\delta X^{\rho}(u)} \frac{\delta}{\delta X_{\nu]}(z)} .
\end{aligned}
$$

Apparently, this $L_{\mu \nu}$ is not primitive and this means that the $U(\mathcal{P})$ is twisted. We give several remarks about this twisted Poincaré symmetry read from (5.4), (5.5):

1. The twisting of $U(\mathcal{P})$ is only due to the second twist $\mathcal{F}_{B}$. This guarantees that a single local vertex operator : $F$ : in $\left(\hat{\mathcal{A}}_{0}\right)_{\mathcal{F}_{B}}$ is still Poincaré covariant after the twist, and thus the spacetime meaning of a vertex operator is unchanged. 
2. The twisting is not closed within $U(\mathcal{P})$, but the additional terms in (5.4) are in $U(\mathfrak{C}) \otimes U(\mathfrak{C})$, and similarly for (5.5). This shows that the twisting does not mix the Lorentz generator with other diffeomorphisms even for products of vertex operators. Therefore, from the spacetime point of view, a Poincaré transformation on products of fields is still a global transformation even after the twist.

3. The twisted product $* \mathcal{F}_{B}$ of two vertex operators reduces to the product of corresponding spacetime fields, but the latter depends on the representations (spins) of $U(\mathcal{P})$, because $\mathcal{F}_{B}$ depends on the Green function $G_{B}$. For instance, the twisted product of two gravitons is different from that of two tachyons due to this fact. Similarly, the Lorentz transformation law for the product of two spacetime fields depends on their spins.

To summarize, from the decomposition of the twist element $\mathcal{F}_{1}=\mathcal{F}_{B} \mathcal{F}_{0}$, we obtain a description of a spacetime with a $B$-field background, such that the effect of background $B$-field is hidden in the twist element $\mathcal{F}_{B}$, while the other matter fields acquires a deformation caused by this twist element. This description of spacetime is valid for closed strings as well, and is different from the description formulated on a commutative spacetime with a matter field $B_{\mu \nu}$ or from the description using a noncommutative space as in the effective theory of open string. Although it is not clear yet how this new description translates into the effective field theory language, our method clearly demonstrates how a background field (other than the metric) can be incorporated into a formulation of a stringy spacetime.

\subsection{Twisted diffeomorphism}

Here we discuss about the twisted diffeomorphism in the context of successive twists. For simplicity, we fix a twist element $\mathcal{F}_{0}$ to be Minkowski background, but the following argument can be applied to any fixed $\mathcal{F}_{0}$ of the form (3.3).

Let $\xi \in \mathfrak{X}$ be a functional vector field of some target space vector field. Its finite version $u=e^{\xi} \in \mathcal{H}=U(\mathfrak{X})$ is group-like $\Delta(u)=u \otimes u$. It acts on the coordinate functional as $u \triangleright X^{\mu}(z)=X^{\mu}(z)+\xi^{\mu}[X(z)]+\mathcal{O}\left(\xi^{2}\right) \equiv Y^{\mu}(z)$. Thus the action of $u$ is interpreted as a coordinate transformation (from the passive viewpoint). Classically, it is a automorphism of the algebra of functionals $u \triangleright(F \cdot G)=F_{u} \cdot G_{u}$, where $F_{u}[X]=(u \triangleright F)[X]=F[Y]$ is understood as a new

functional written in the transformed coordinates. However, this is not the case for $\mathcal{A}_{\mathcal{F}_{0}}$, that is seen by rewriting the action of $u$ on the twisted product

$$
u \triangleright\left(F *_{\mathcal{F}_{0}} G\right)=m \circ \mathcal{F}_{0}^{-1} \Delta_{\mathcal{F}_{0}}(u) \triangleright(F \otimes G)=m \mathcal{F}_{1}^{-1} \triangleright(u \triangleright F \otimes u \triangleright G)=F_{u} *_{\mathcal{F}_{1}} G_{u} .
$$

Namely, from the passive viewpoint, the product is also needed to be transformed to give a new twist element [1]

$$
\mathcal{F}_{1}=(u \otimes u) \mathcal{F}_{0} \Delta\left(u^{-1}\right) .
$$

Note that (5.6) is also a coboundary twist since it can be written in the form (3.4) with the new normal ordering $\mathcal{N}_{1}=u \mathcal{N}_{0} u^{-1}$. As a consequence, there are two vertex operator algebras $\hat{\mathcal{A}}_{0}$ and $\hat{\mathcal{A}}_{1}$ and they are related by the $u$-action as ${ }_{\circ}^{\circ} F_{u_{\circ}^{\circ}}=\mathcal{N}_{1} u \triangleright F=u \mathcal{N}_{0} \triangleright F=u \triangleright: F:$. It is natural that the vertex operator algebra transforms under the diffeomorphism, because the change of the background needs a new mode expansion in the operator formulation. However, $u \triangleright: F$ : is not well-defined as operators since $u$ is not an element in $\hat{\mathcal{H}}_{0}$. In other words, $u \triangleright: F$ : contains formal divergent terms. See [1] for more details to come to this point. Here we would like to discuss that this difficulty is solved by applying the method in Section 4 .

To this end, we will first decompose $\mathcal{F}_{1}(5.6)$ to the form $\mathcal{F}_{1}=\mathcal{F}_{d} \mathcal{F}_{0}$. It is easy to show that

$$
\mathcal{F}_{d}=(u \otimes u) \Delta_{\mathcal{F}_{0}}\left(u^{-1}\right)
$$


is the desired twist element in $\mathcal{H}_{\mathcal{F}_{0}}$. Note that (5.7) is not in $U(\mathfrak{C}) \otimes U(\mathfrak{C})$ in general, nor of the type (3.3). By applying the rule (4.6), the deformation of the vertex operator algebra $\hat{\mathcal{A}}_{0}$ to $\left(\hat{\mathcal{A}}_{0}\right)_{\tilde{\mathcal{F}}_{d}}$ is governed by

$$
\begin{aligned}
\tilde{\mathcal{F}}_{d} & =\left(\mathcal{N}_{0} \otimes \mathcal{N}_{0}\right) \mathcal{F}_{d}\left(\mathcal{N}_{0}^{-1} \otimes \mathcal{N}_{0}^{-1}\right)=\left(\mathcal{N}_{0} \otimes \mathcal{N}_{0}\right)(u \otimes u) \mathcal{F}_{0}\left(u^{-1} \otimes u^{-1}\right) \mathcal{F}_{0}^{-1}\left(\mathcal{N}_{0}^{-1} \otimes \mathcal{N}_{0}^{-1}\right) \\
& =\left(\mathcal{N}_{0} \otimes \mathcal{N}_{0}\right)(u \otimes u)\left(\mathcal{N}_{0}^{-1} \otimes \mathcal{N}_{0}^{-1}\right) \Delta\left(\mathcal{N}_{0}\right) \Delta\left(u^{-1}\right) \Delta\left(\mathcal{N}_{0}^{-1}\right)=(\tilde{u} \otimes \tilde{u}) \Delta\left(\tilde{u}^{-1}\right) .
\end{aligned}
$$

This does not reduce to 1 because $\tilde{u}=\mathcal{N}_{0} u \mathcal{N}_{0}^{-1} \in \hat{\mathcal{H}}_{0}$ is not group-like. Thus the twisting by $\tilde{\mathcal{F}}_{d}$ must deform the operator product. In $\left(\hat{\mathcal{A}}_{0}\right)_{\tilde{\mathcal{F}}_{d}}$, a single vertex operator and a product of two vertex operators are related to that of $\hat{\mathcal{A}}_{0}$ as $: F_{u}:=\tilde{u} \triangleright: F:$ and

$$
\begin{aligned}
: F_{u}: *_{\tilde{\mathcal{F}}_{d}}: G_{u}: & =m \circ \tilde{\mathcal{F}}_{d}^{-1} \triangleright\left(: F_{u}: \otimes: G_{u}:\right)=m \circ \Delta(\tilde{u})\left(\tilde{u}^{-1} \otimes \tilde{u}^{-1}\right) \triangleright\left(: F_{u}: \otimes: G_{u}:\right) \\
& =\tilde{u} \triangleright(: F:: G:),
\end{aligned}
$$

which are given by well-defined actions of $\tilde{u}$.

We conclude that any background diffeomorphic to the original background $\mathcal{F}_{0}$ is also described by a twist $\mathcal{F}_{1}$ (5.6). In the vertex operator language, this causes a deformation of the original OPE, without changing the background itself. We can now see the effect of diffeomorphism by comparing $\hat{\mathcal{A}}_{0}$ and $\left(\hat{\mathcal{A}}_{0}\right)_{\tilde{\mathcal{F}}_{d}}$ instead of $\hat{\mathcal{A}}_{0}$ and $\hat{\mathcal{A}}_{1}$.

\subsection{Zero modes}

In this section, we discuss some results about the role of the zero modes in twist quantization. Let us fix a twist element $\mathcal{F}_{0}$ for the Minkowski background, which is given by the Green function (3.11). Our convention on the VEV defined in (3.2) for $\hat{\mathcal{A}}_{0}$ has the properties

$$
\langle 1\rangle_{0}=1, \quad\left\langle X^{\mu}(z)\right\rangle_{0}=0, \quad\left\langle: e^{i k \cdot X(z)}:\right\rangle_{0}=1 .
$$

These do not match with the conventional correlation functions, that have momentum conservation in target spacetime ${ }^{3}$. Here we argue that this difficulty can be removed easily by changing the constant term in the propagator, or equivalently, a further twisting on the zero modes.

Motivated by a tachyon one point function satisfying the momentum conservation

$$
\left\langle: e^{i k \cdot X(z)}:\right\rangle \propto(2 \pi)^{d} \delta^{(d)}(k),
$$

we define a map $\tilde{\tau}: \mathcal{A} \rightarrow \mathbb{C}$ by

$$
\tilde{\tau}(F[X]):=\left.\int d^{d} x F[X]\right|_{X=x},
$$

that is, we set $X^{\mu}(z)=x^{\mu}$ in the functional instead of setting $X=0$ as in (3.2) and then integrate over $x$. This is a consistent treatment comparing with the path integral quantization. There, since the zero modes $x^{\mu}$ of $X^{\mu}(z)$ do not appear in the action, they are integrated out separately from non-zero modes. (5.9) corresponds to this integration. Note that the integrand in (5.9) is related to $\tau$ as $\left.F[X]\right|_{X=x}=\tau(F[X+x])=\tau\left(e^{-i x^{\mu} P_{\mu}} \triangleright F[X]\right)$, where $P_{\mu} \in U(\mathfrak{C})$ is the translation generator in (2.6). If we use a following relation as an operator ${ }^{4}$

$$
\int d^{d} x e^{-i x^{\mu} P_{\mu}}=(2 \pi)^{d} \delta^{(d)}(P)=(2 \pi)^{d} \lim _{C \rightarrow \infty}\left(\frac{C}{2 \pi}\right)^{\frac{d}{2}} e^{-\frac{C}{2} \eta^{\mu \nu} P_{\mu} P_{\nu}},
$$

\footnotetext{
${ }^{3}$ They are conventional correlation functions as a two dimensional scalar field theory.

${ }^{4}$ The Wick rotation should be performed in the time direction.
} 
then the map $\tilde{\tau}$ is also written as

$$
\tilde{\tau}(F[X])=\lim _{C \rightarrow \infty}(2 \pi C)^{\frac{d}{2}} \tau\left(\mathcal{N}_{d}^{-1} \triangleright F[X]\right),
$$

where we defined a Hopf algebra element $\mathcal{N}_{d} \in U(\mathfrak{C})$ by

$$
\mathcal{N}_{d}=e^{\frac{C}{2} \eta^{\mu \nu} P_{\mu} P_{\nu}} .
$$

In the following, $C$ is always understood as a positive divergent constant. For example,

$$
\tilde{\tau}(1)=(2 \pi C)^{d / 2}, \quad \tilde{\tau}\left(e^{i k \cdot X(z)}\right)=(2 \pi)^{d} \delta^{(d)}(k) .
$$

By renormalizing the overall factor, we define a new map (with suffix $d$ ) as

$$
\tau_{d}(F[X])=\tau\left(\mathcal{N}_{d}^{-1} \triangleright F[X]\right) .
$$

For example,

$$
\tau_{d}(1)=1, \quad \tau_{d}\left(e^{i k \cdot X(z)}\right)=\frac{(2 \pi)^{d} \delta^{(d)}(k)}{(2 \pi C)^{d / 2}} .
$$

The divergent overall factor $(2 \pi C)^{d / 2}$ in (5.11) comes form the infinite volume $\sim \delta^{d}(0)$ in the target space. Thus the relation between the two maps $\tilde{\tau}$ and $\tau_{d}$ is exactly the same as that between the unnormalized and normalized path integrals.

This new definition of the VEV (5.12) suggests a relation to a new twisting. We define $\mathcal{F}_{1}$ of the form (3.3) with $G_{1}$ given by adding a (divergent) constant term $C$ as

$$
G_{1}^{\mu \nu}(z, w)=G_{0}^{\mu \nu}(z, w)+C \eta^{\mu \nu} .
$$

This modification is, in fact, needed for the compact worldsheet [10]. When calculating the Feynman propagator on the worldsheet, such a divergent term in the Green function appears as an IR cut off, sometimes written as $G_{1}^{\mu \nu}(z, w)=-\alpha^{\prime} \eta^{\mu \nu} \ln \mu|z-w|(0<\mu)$, thus $C=-\alpha^{\prime} \ln \mu$. From the target space point of view, the term reflects the non-compactness of the Minkowski spacetime (i.e., zero mode part $\left\langle 0\left|\hat{x}^{\mu} \hat{x}^{\nu}\right| 0\right\rangle$ in the two point function).

We can now apply the decomposition method to the twist $\mathcal{F}_{1}=\mathcal{F}_{d} \mathcal{F}_{0}$ and the normal ordering $\mathcal{N}_{1}=\mathcal{N}_{d} \mathcal{N}_{0}$, where $\mathcal{N}_{d}$ is defined in (5.10) and

$$
\mathcal{F}_{d}=e^{C \eta^{\mu \nu} P_{\mu} \otimes P_{\nu}}
$$

but in the present case it is more appropriate to take the first twist by $\mathcal{F}_{d}$, and then to perform the second twisting by $\mathcal{F}_{0}$. This leads to a structure similar to (4.8) as

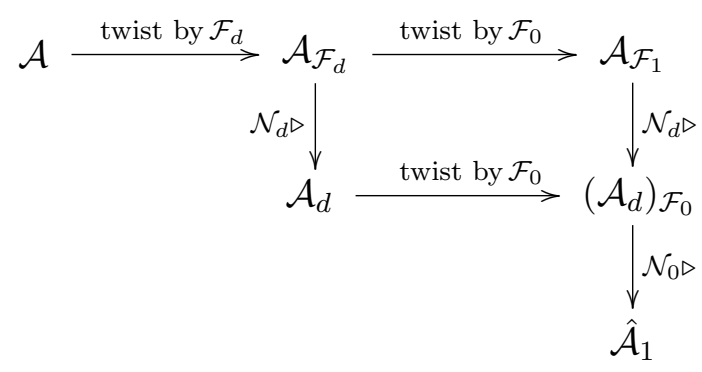

In this diagram (5.13), we denote the "normal ordered" module algebra of the first twist $\mathcal{F}_{d}$ as $\mathcal{A}_{d}$ (not as $\hat{\mathcal{A}}_{d}$ ), since the first twist by $\mathcal{F}_{d}$ is not regarded as a quantization. This is because $\mathcal{N}_{d}$ acts only on the zero mode part of functionals $F[X]$ and it does not cause any divergence coming 
from coincident points. For example, $\mathcal{N}_{d} \triangleright \partial_{a} X^{\mu}(z) e^{i k \cdot X(z)}=e^{\frac{C}{2} k^{2}} \partial_{a} X^{\mu}(z) e^{i k \cdot X(z)}$ behaves as a classical functional. Correspondingly, we prefer to write an element $\mathcal{A}_{d} \ni F_{d}$ instead of $\mathcal{N}_{d} \triangleright F$. The map $\mathcal{A}_{d} \rightarrow \mathbb{C}$ induced by $\tau: \mathcal{A} \rightarrow \mathbb{C}$ also does not have an interpretation as a VEV, but it is the map $\tau_{d}$ given in (5.12). Indeed, we have a relation $\tau_{d}\left(F_{d}\right)=\tau\left(\mathcal{N}_{d}^{-1} \mathcal{N}_{d} \triangleright F\right)=\tau(F)$ as well as $\tau_{d}\left(F_{d} G_{d}\right)=\tau(F G)$.

Then, if we regard $\mathcal{A}_{d}$ as a classical module algebra of functionals, the second twist by $\mathcal{F}_{0}$ (a sub-diagram staring from $\mathcal{A}_{d}$ ) is interpreted as a quantization. In particular, the VEV $\left(\mathcal{A}_{d}\right)_{\mathcal{F}_{0}} \rightarrow \mathbb{C}$ is defined like (3.2) but based on $\tau_{d}$ (not on $\tau$ ). This is the main effect of the first twist $\mathcal{F}_{d}$, which implies the momentum conservation. The vertex operator algebra is now given by $\hat{\mathcal{A}}_{1}$, whose elements have the form $: F_{d}$ :, and the relation to $\left(\mathcal{A}_{d}\right)_{\mathcal{F}_{0}}$ is as usual:

$$
\tau_{d}\left(F_{d}\right)=\tau_{d}\left(\mathcal{N}_{0}^{-1}: F_{d}:\right)=\left\langle: F_{d}:\right\rangle_{1}, \quad \tau_{d}\left(F_{d} * \mathcal{F}_{0} G_{d}\right)=\left\langle: F_{d}:: G_{d}:\right\rangle_{1} .
$$

Note the subscript 1 in the $\operatorname{VEV}\langle\cdots\rangle_{1}$. It depends both on $\mathcal{N}_{0}^{-1}$ (the quantization) and $\tau_{d}$ (the chosen vacuum state). Of course, they equal to $\tau(F)$ and $\tau\left(F *_{\mathcal{F}_{1}} G\right)$, respectively.

As an example, an ordinary tachyon $n$-point correlation function is given by setting each local functional $F_{d}[X]=e^{i k X(z)}$ in $\mathcal{A}_{d}$ as

$$
\begin{aligned}
& \left\langle: e^{i k_{1} X\left(z_{1}\right)}: \cdots: e^{i k_{n} X\left(z_{n}\right)}:\right\rangle_{1}=\tau_{d}\left(e^{i k_{1} X\left(z_{1}\right)} *_{\mathcal{F}_{0}} \cdots * \mathcal{F}_{0} e^{i k_{n} X\left(z_{n}\right)}\right) \\
& \quad=\tau\left(e^{-\frac{C}{2} k_{1}^{2}} e^{i k_{1} X\left(z_{1}\right)} *_{\mathcal{F}_{1}} \cdots *_{\mathcal{F}_{1}} e^{-\frac{C}{2} k_{1}^{2}} e^{i k_{n} X\left(z_{n}\right)}\right) \\
& \quad=e^{-\frac{C}{2} \sum_{i} k_{i}^{2}-C \sum_{i<j} k_{i} \cdot k_{j}+\alpha^{\prime} \sum_{i<j} k_{i} \cdot k_{j} \ln \left|z_{i}-z_{j}\right|}=\frac{(2 \pi)^{d} \delta^{(d)}\left(\sum_{i} k_{i}\right)}{(2 \pi C)^{d / 2}} \prod_{i<j}\left|z_{i}-z_{j}\right|^{\alpha^{\prime} k_{i} \cdot k_{j}},
\end{aligned}
$$

which is equivalent to starting with local functionals $F[X]=e^{-\frac{C}{2} k^{2}} e^{i k X(z)}$ in $\mathcal{A}$. This example shows that all the constant $C$ appearing in the intermediate expression are cancelled among them except for the overall normalization, and the latter is also cancelled with the momentum delta-function in the case of a momentum conserving correlation function. Thus for renormalized functionals $F_{d}$, it is designed so as to give a well-defined vacuum expectation values. On the other hand, this example also suggests that a tachyon field $T(x)$ in spacetime corresponds to an already renormalized functional $F_{d}[X]=e^{i k X(z)}$ on the worldsheet. At this point, we have not yet fully understood the rule to give such a correspondence between spacetime fields and worldsheet functionals. Since it needs both worldsheet and spacetime analysis, we leave it for a further study.

In summary, the quantization with the momentum conservation is simply realized by starting with renormalized classical functionals $\mathcal{A}_{d} \in F_{d}$, and by quantizing it with respect to the twist $\mathcal{F}_{0}$ and the map $\tau_{d}$. On the other hand, this quantiztion is equivalent to the conbined twist $\mathcal{F}_{1}$ (and the map $\tau$ ) from the point of view of the original functionals $\mathcal{A}$.

Let us now focus on the symmetry. Of course, there is the same kind of structure as (5.13) among corresponding Hopf algebras. It can be shown that the Poincaré-Hopf algebra $U(\mathcal{P})$ is a twist invariant Hopf subalgebra of both $\mathcal{H}_{d}$ and $\left(\mathcal{H}_{d}\right)_{\mathcal{F}_{0}}$. This is proved in the same way as was done in [1] for the twist $\mathcal{F}_{0}$, because only the following properties of the Green function $G$ are used: $G^{\mu \nu}$ is proportional to $\eta^{\mu \nu}$, and it is symmetric $G^{\mu \nu}(z, w)=G^{\nu \mu}(w, z)$. Therefore, $U(\mathcal{P})$ remains algebraically a true symmetry under any step in $(5.13)$. On the other hand, the violation of the momentum conservation in the original quantization (5.8) is due to the bad choice of a map $\tau$, or equivalently the choice of a translation non-invariant vacuum state.

The argument in this subsection seems to indicate that the diffeomorphism or the $B$-field background acquire some effect from the extra normal ordering $\mathcal{N}_{d}$ in the zero mode structure and in the effective theory. We hope to come back to this point in the future reference. 


\section{Discussion}

As we have argued, one of advantages of the twist quantization is that there is a direct relation between quantization and symmetry via the Hopf algebra. One typical example is the Poincaré symmetry $U(\mathcal{P})$ which is twisted by $\mathcal{F}_{1}$ in a $B$-field background. In order to compare the twist quantization with known formulations, we formulated a useful method to decompose the twist into two successive twists. It is then applied to several examples, $B$-field background, finite twisted diffeomorphism, and zero modes.

All the example of twist elements so far are coboundary, that are cohomologically trivial. However, the decomposition is rather arbitrary and possible as far as the second twist satisfies the cocycle condition under the first twist, the condition corresponding to (4.2). Therefore, the method proposed in this paper can in principle be applied to more non-trivial backgrounds, such as non-constant metric or $B$-field. For this, it is natural to ask whether the quantization in all the backgrounds can be written as twist quantization, and if so, wheather the corresponding twist elements are coboundary. We think naively that any background corresponds to a quantization with a coboundary twist, since our star-products are the time ordered product, which is starequivalent to the ordinary commutative product, but this has still to be proven. The further study along this line would help to get a better understanding of the structure of the stringy geometry. Another important and interesting open question is the relation between a twist element for a generic background and the conformal symmetry.

\section{A Proof for successive twists}

We show two relations $\left(\Delta_{\mathcal{F}_{0}}\right)_{\mathcal{F}_{d}}=\Delta_{\mathcal{F}_{1}}$ and $\left(S_{\mathcal{F}_{0}}\right)_{\mathcal{F}_{d}}=S_{\mathcal{F}_{1}}$ for successive twists (4.3). The first equation is easily shown by definition $\Delta_{\mathcal{F}_{1}}(h)=\mathcal{F}_{1} \Delta(h) \mathcal{F}_{1}^{-1}=\mathcal{F}_{d} \mathcal{F}_{0} \Delta(h) \mathcal{F}_{0}^{-1} \mathcal{F}_{d}^{-1}=$ $\left(\Delta_{\mathcal{F}_{0}}\right)_{\mathcal{F}_{d}}(h)$ for $\forall h$. To show the second equation, we recall that the definition $S_{\mathcal{F}}(h)=$ $U S(h) U^{-1}$. By using the notation $\mathcal{F}=f^{\alpha} \otimes f_{\alpha}$ and $\mathcal{F}^{-1}=\bar{f}^{\alpha} \otimes \bar{f}_{\alpha}$, operators $U, U^{-1} \in \mathcal{H}$ is written as $U=\mu(\mathrm{id} \otimes S) \mathcal{F}=f^{\alpha} S\left(f_{\alpha}\right)$, and similarly, $U^{-1}=\mu(S \otimes \mathrm{id}) \mathcal{F}_{12}^{-1}=S\left(\bar{f}_{\alpha}\right) \bar{f}^{\alpha}$. We denote three $U$ 's corresponding to $\mathcal{F}_{0}, \mathcal{F}_{d}$ and $\mathcal{F}_{1}$ as $U_{0}, U_{d}$, and $U_{1}$, respectively. Then by using $\mathcal{F}_{1}=$ $\mathcal{F}_{d} \mathcal{F}_{0}$, we can rewrite $U_{1}$ as $U_{1}=\mu(\mathrm{id} \otimes S) \mathcal{F}_{1}=f_{1}^{\alpha} S\left(f_{1 \alpha}\right)=f_{d}^{\alpha} f_{0}^{\beta} S\left(f_{0 \beta}\right) S\left(f_{d \alpha}\right)=f_{d}^{\alpha} U_{0} S\left(f_{d \alpha}\right)=$ $\mu\left(1 \otimes U_{0}\right)(\mathrm{id} \otimes S) \mathcal{F}_{d}=\left[\mu\left(1 \otimes U_{0}\right)(\mathrm{id} \otimes S) \mathcal{F}_{d}\left(1 \otimes U_{0}^{-1}\right)\right] \cdot U_{0}=\left[\mu\left(\mathrm{id} \otimes S_{\mathcal{F}_{0}}\right) \mathcal{F}_{d}\right] \cdot U_{0}=U_{d} \cdot U_{0}$. Note that $U_{d} \in \mathcal{H}_{\mathcal{F}_{0}}$. Therefore, $S_{\mathcal{F}_{1}}(h)=U_{1} S(h) U_{1}^{-1}=U_{d} U_{0} S(h) U_{0}^{-1} U_{d}^{-1}=U_{d} S_{\mathcal{F}_{0}}(h) U_{d}^{-1}=$ $\left(S_{\mathcal{F}_{0}}\right)_{\mathcal{F}_{d}}(h)$.

\section{Acknowledgements}

The authors would like to thank M. Mori for collaboration and useful discussions. We also thank to Dr. U. Carow-Watamura for useful comments and discussions. This work is supported by Grant-in-Aid for Scientific Research from the Ministry of Education, Culture, Sports, Science and Technology, Japan, No. 19540257.

\section{References}

[1] Asakawa T., Mori M., Watamura S., Hopf algebra symmetry and string theory, Progr. Theoret. Phys. 120 (2008), 659-689, arXiv:0805.2203.

[2] Asakawa T., Mori M., Watamura S., Twist quantization of string and B field background, J. High Energy Phys. 2009 (2009), no. 4, 117, 25 pages, arXiv:0811.1638.

[3] Drinfeld V.G., Quasi-Hopf algebras, Leningrad Math. J. 1 (1990), 1419-1457.

[4] Chaichian M., Kulish P.P., Nishijima K., Tureanu A., On a Lorentz-invariant interpretation of noncommutative space-time and its implications on noncommutative QFT, Phys. Lett. B 604 (2004), 98-102, hep-th/0408069. 
[5] Koch F., Tsouchnika E., Construction of $\theta$-Poincaré algebras and their invariants on $\mathcal{M}_{\theta}$, Nuclear Phys. B 717 (2005), 387-403, hep-th/0409012.

[6] Aschieri P., Blohmann C., Dimitrijević M., Meyer F., Schupp P., Wess J., A gravity theory on noncommutative spaces, Classical Quantum Gravity 22 (2005), 3511-3532, hep-th/0504183.

[7] Watamura S., Noncommutative geometry in string and twisted Hopf algebra of diffeomorphism, Gen. Relativity Gravitation, to appear.

[8] Aschieri P., Lizzi F., Vitale P., Twisting all the way: from classical mechanics to quantum fields, Phys. Rev. D $\mathbf{7 7}$ (2008), 025037, 16 pages, arXiv:0708.3002.

[9] Majid S., Foundations of quantum group theory, Cambridge University Press, Cambridge, 1995.

[10] Polchinski J., String theory, Cambridge University Press, Cambridge, 1998.

[11] Álvarez-Gaumé L., Meyer F., Vázquez-Mozo M.A., Comments on noncommutative gravity, Nuclear Phys. B 753 (2006), 92-117, hep-th/0605113.

[12] Fradkin E.S., Tseytlin A.A., Nonlinear electrodynamics from quantized strings, Phys. Lett. B 163 (1985), $123-130$.

[13] Callan C.G., Lovelace C., Nappi C.R., Yost S.A., String loop corrections to beta functions, Nuclear Phys. B 288 (1987), 525-550.

[14] Abouelsaood A., Callan C.G., Nappi C.R., Yost S.A., Open strings in background gauge fields, Nuclear Phys. B 280 (1987), 599-624.

[15] Braga N.R.F., Carrion H.L., Godinho C.F.L., Normal ordering and boundary conditions in open bosonic strings, J. Math. Phys. 46 (2005), 062302, 5 pages, hep-th/0412075.

[16] Braga N.R.F., Carrion H.L., Godinho C.F.L., Normal ordering and boundary conditions for fermionic string coordinates, Phys. Lett. B 638 (2006), 272-274, hep-th/0602212.

[17] Chakraborty B., Gangopadhyay S., Hazra A.G., Normal ordering and noncommutativity in open bosonic strings, Phys. Rev. D $\mathbf{7 4}$ (2006), 105011, 6 pages, hep-th/0608065.

[18] Gangopadhyay S., Hazra A.G., Normal ordering and non(anti)commutativity in open super strings, Phys. Rev. D 75 (2007), 065026, 6 pages, hep-th/0703091.

[19] Seiberg N., Witten E., String theory and noncommutative geometry, J. High Energy Phys. 1999 (1999), no. 9, 032, 93 pages, hep-th/9908142.

[20] Oeckl R., Untwisting noncommutative $\mathbb{R}^{d}$ and the equivalence of quantum field theories, Nuclear Phys. B $\mathbf{5 8 1}$ (2000), 559-574, hep-th/0003018.

[21] Watts P., Derivatives and the role of the Drinfeld twist in noncommutative string theory, hep-th/0003234. 